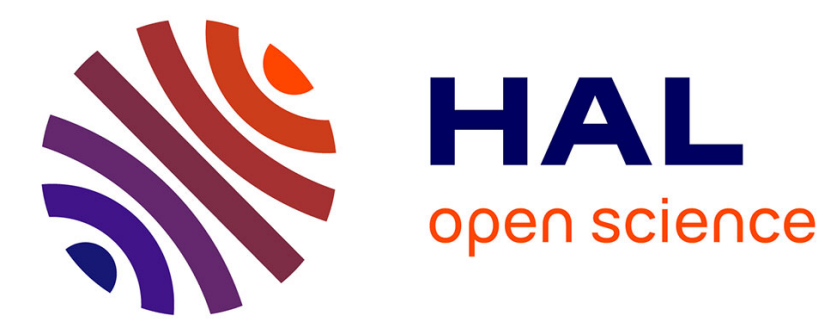

\title{
Parity violation and the nature of charges
}

\author{
B. Desplanques
}

\section{To cite this version:}

B. Desplanques. Parity violation and the nature of charges. 3rd International Workshop: "From parity violation to hadronic structure and more .." (PAVI06), May 2006, Milos island, Greece. pp.533-535, 10.1140/epja/i2006-10431-y . in2p3-00116670

\section{HAL Id: in2p3-00116670 https://hal.in2p3.fr/in2p3-00116670}

Submitted on 27 Nov 2006

HAL is a multi-disciplinary open access archive for the deposit and dissemination of scientific research documents, whether they are published or not. The documents may come from teaching and research institutions in France or abroad, or from public or private research centers.
L'archive ouverte pluridisciplinaire HAL, est destinée au dépôt et à la diffusion de documents scientifiques de niveau recherche, publiés ou non, émanant des établissements d'enseignement et de recherche français ou étrangers, des laboratoires publics ou privés. 


\title{
Parity violation and the nature of charges
}

\author{
B. Desplanques \\ Laboratoire de Physique Subatomique et de Cosmologie (UMR CNRS/IN2P3-UJF-INPG), \\ F-38026 Grenoble Cedex, France
}

Received: date / Revised version: date

\begin{abstract}
The origin of parity violation in physics is still unknown. At the present time, it is introduced in the theory by requiring that the $S U(2)$ subgroup entering the description of interactions involves the left components. In the present contribution, one elaborates upon a suggestion made by Landau that particles and antiparticles could be like "stereo-isomeric" molecules, which would naturally provides parity violation. Particles and antiparticles could thus be combinations of the parity doublets associated with a chiral symmetry realized in the Wigner-Weyl mode. Consequences of such a description and the possible problems it could raise are examined.
\end{abstract}

PACS. 11.30.Er Charge conjugation, parity, time reversal and other discrete symmetries

\section{Introduction}

Thinking about the $\theta-\tau$ puzzle, Lee and Yang were led to suggest, 50 years ago, that the parity symmetry could $\checkmark$ be violated in weak interactions [1]. This was confirmed a , few months later by $\mathrm{Wu}$ et al. who observed a preferential direction for the $\beta$ emission in the decay of oriented ${ }^{60} \mathrm{Co}[2]$. Since then, the knowledge of the weak interaction has considerably increased. Together with strong and electromagnetic interactions, it is embedded in the standard $\checkmark 5$ model, which is based on the $S U(3)_{c} \otimes S U(2)_{L} \otimes U(1)_{Y}$ gauge group with spontaneous symmetry breaking. Parity $\checkmark$ violation is ascribed to the appearance of the left components associated with the $S U(2)$ weak isospin subgroup $\left(S U(2)_{L}\right)$. It is sometimes considered that this property could be a low energy one and that the standard model should be completed by another $S U(2)$ subgroup, involving this time the right components $\left(S U(2)_{R}\right)$ [3]. Implying further higher mass gauge bosons, the parity symmetry could thus be restored in high-energy processes.

When trying to answer some question, it is not rare that one has better to enlarge the problem. Another question, which is also a fundamental one, is the nature of charges or, for our concern here, what makes a particle different from its antiparticle. At present, particles and $\exists$ antiparticles appear as solutions of the same equation, the Dirac one for instance, and they are essentially characterized by some numbers, reflecting their "charges".

Could it be that parity violation and the nature of charges be related to each other? A hint is provided by the approximate conservation of the $P C$ symmetry, which suggests that the two operations $P$ and $C$ have a deep relationship. Which one however?

Some ideas along the above lines have been briefly proposed by Landau [4] soon after the suggestion of the parity violation by Lee and Yang. He was considering that the difference between particles and antiparticles, as far as the space symmetry is concerned, "is no greater than that due to chemical stereo-isomerism". Their "charges" of interest here are especially the leptonic and baryonic ones.

In the present paper, we want to elaborate upon these ideas. In sect. 2 , we make a couple of observations that relativize to some extent the origin of parity violation in physics. Section 3 is devoted to describing a framework accounting for particles and antiparticles. The fourth section is concerned with the origin of parity violation in this framework and the emergence of the charge conjugation as a parity operation on an internal structure with a chiral character. Some results from a toy model are presented in sect. 5. An outlook is given in sect. 6 .

\section{Some observations}

When asked about the origin of parity violation in physics, an answer often given is that nature is essentially lefthanded. This is built in the theory which relies on the gauge subgroup $S U(2)_{L}$. Had the theory been written in terms of the charge conjugate fields $(\psi$ describes the creation of a particle and the destruction of the antiparticle, instead of the inverse), the gauge subgroup would be $S U(2)_{R}$. The left-handedness would have transformed into a right-handedness. The handedness thus depends on what we refer to as particles or antiparticles. Considering both particles and antiparticles on the same footing, there would not be any preferred handedness. Actually, this symmetry is nothing but the $P C$ symmetry which we assume to hold here. It suggests that the charge conjugation operation, $C$, could have something to do with a usual parity operation. 
Another observation concerns the mathematical definition of a parity operation. As noticed by Lee and Wick [5], if $P$ is a parity operation, the product of $P$ by any unitary operator $U$ that leaves the interaction invariant is also a parity operation. This can be applied to the case where one takes for $U$ the charge conjugation operator $C$, which leaves invariant the strong and electromagnetic interactions. Thus, the product $P C$ could be considered as a parity operation. This definition is quite advantageous as the corresponding symmetry essentially holds for all interactions. Again, this feature suggests that the charge conjugation could have something to do with a usual parity operation.

Following Landau and anticipating on next sections, we will speak of the product $P C$ as a combined parity operation. We will denote it as $P C=\mathcal{P}$, to remind that the operations $P$ and $C$ originate from a unique parity operation, acting however on different degrees of freedom.

\section{Framework for particles and antiparticles}

Models with chiral symmetry and parity symmetry have received a lot of attention in the strong interaction domain (QCD). In the simplest case and quite generally, they are characterized by a conserved current, $J_{\mu}^{A}(x)$, and an anticommutation relation between the axial charge $Q^{A}=$ $\int d \boldsymbol{x} J_{0}^{A}(x)$, and the (genuine) parity operator, $\mathcal{P}$ :

$$
\left[\mathcal{P}, Q^{A}\right]_{+}=\mathcal{P} Q^{A}+Q^{A} \mathcal{P}=0 .
$$

A first realization of the symmetry, which stems from the above equation in the case $Q^{A} \mid$ state $\neq 0>\neq 0$, assumes that particles appear in the form of doublets: two equalmass states of opposite parity or, equivalently, two states with opposite chiral charge, transforming into each other by a parity operation (Wigner-Weyl mode). It supposes $Q^{A} \mid 0>=0$. A second realization (Nambu-Jona-Lasinio mode) supposes $Q^{A} \mid 0>\neq 0$ and implies the existence of a Goldstone boson. In absence of parity doublets, this is the realization that has been retained in the QCD case where the Goldstone boson is known to be the pion. While looking for parity doublets, it has been assumed that the charges of these particles should be the same. Little attention has been given to the fact that they could be different and have a chiral character. Thus, particles and antiparticles could be associated to the states with opposite chiral charges of an interaction exhibiting chiral symmetry or as combinations of the parity doublets it implies. It is evident that this chiral symmetry has nothing to do with the QCD one but the existence of this one makes plausible the existence of an other one in a different sector of the interaction.

\section{Origin of parity violation and $C$ operation}

In atomic or nuclear physics, most of the observed parityviolating effects are ascribed to some parity admixture in the systems one is dealing with. The question arises whether it could also be so at the more fundamental level. In the present case, an important observation about parity conservation stems from eq. (1). The parity operation $\mathcal{P}$ and the chiral charge $Q^{A}$ do not commute; one cannot therefore generally have eigenstates of the parity and the chiral charge simultaneously. As most experiments involve "charged" particles in the initial or final states, one should expect to see some apparent parity violation, though parity is conserved at the interaction level. The observed parity violation would reflect the fact that these particles are not, intrinsically, eigenstates of the parity.

While parity violation could be traced back to charges with a chiral nature, the problem would rather be to explain why it is so strong in some cases $(100 \%$ in weak interactions) and absent in other cases (strong and electromagnetic interactions). An answer would require to consider some dynamics, perhaps unknown at the present time. A qualitative understanding can nevertheless be proposed. It supposes that the spin and momentum properties of the chiral substructure decouple of those of the particle as a whole. In this decoupling limit, it is conceivable that the genuine parity operation $\mathcal{P}$ factorizes into a part involving the external degrees of freedom (the usual $P$ operation) and a part involving the internal degrees of freedom (the usual $C$ operation). Of course, the decoupling cannot be complete and some parity violation reflecting the chiral nature of "charges" could show up. It is noticed that some experiments do not necessarily involve "charged" particles, like the neutral kaon decay into two mesons (the $\pi^{+} \pi^{-}$state is globally considered here as a neutral one). Parity violation in this case could be observed too. Actually, this would be a true parity violation, which is known as a $P C$ violation [6].

The above explanation ascribing parity violation to an intrinsic chiral structure goes beyond Landau's proposal, which was likely to be only suggestive. Relying on some underlying chiral symmetry like here makes it more systematic, as implied by observation and accounted for by the standard model.

Developments presented in this section have a rather general character. They do not however provide any realistic description of the physical world. Actually, without entering much into details, many questions may be raised. The first one concerns the nature of the chiral structure that could underly some charges and their quantization (possibly approximate). A second non-trivial question has to do with the conservation of the axial current that stems from the chiral symmetry we assumed, taking into account that particles have a mass. Other questions involve the dynamics. How this one makes the spin and momentum properties of the underlying chiral structure to approximately decouple from those of the particle as a whole? or also how particles get mass taking into account that the above chiral symmetry could be realized by a pair of massless particles of opposite helicities (like for a Majorana neutrino). A further but different-type question concerns the equation that could play here the role of the Dirac equation. In the picture developped above, parti- 
cles and antiparticles are somewhat on the same footing, a unique combined parity operation, $\mathcal{P}(=P C)$, transforming one into the other. In the context of the Dirac equation, antiparticles appear as holes in a Fermi sea filled up by negative-energy states.

\section{A toy model}

In order to get some insight on the answers to questions raised in the previous section, but also to emphasize possible problems, we here consider a particular model that is inspired from the Nambu-Jona-Lasinio model [7], but with an essential difference [8]. As we want to associate "charges" to some chiral structure, we change the role of the spin, whose projection along some direction was assumed to be conserved by elementary excitations in this model, with the helicity one.

In a mean-field approximation, it is conceivable that the resulting Lagrangian density reads:

$$
\mathcal{L}(x)=\frac{1}{2}\left(i \bar{\psi}(x) \gamma^{\mu} \partial_{\mu} \psi(x)-m \bar{\psi}(x) \gamma . \epsilon \gamma_{5} \psi(x)\right),
$$

where $\epsilon^{\mu}$ (with $\epsilon^{2}=-1$ ), which represents the polarization carried by the underlying chiral structure, could play a role similar to the deformation one in the case of intrinsically non-spherical nuclei. It is easily checked that the above Lagrangian exhibits some chiral symmetry and that the associated axial current and chiral charge (the helicity number in the present case) read:

$$
J_{\mu}^{A}(x)=\frac{1}{2} \bar{\psi}(x) \gamma_{\mu} \gamma_{5} \psi(x), \quad Q^{A}=\int d \boldsymbol{x} J_{0}^{A}(x) .
$$

In momentum space, it leads to the equation:

$$
\left(\gamma . p-m \gamma . \epsilon \gamma_{5}\right) \psi_{\epsilon}(p)=0 .
$$

Solutions to this last equation are obtained under the condition $\epsilon \cdot p=0$, which corresponds to a decoupling between the spin carried by the underlying chiral structure and the momentum of the system as a whole. They can be expressed in terms of the standard solutions of the Dirac equation:

$$
\begin{aligned}
& \psi_{\epsilon}(p) \propto\left(1+\gamma_{5}\right)(1-\gamma . \epsilon) u(p), \\
& \psi_{\epsilon}(p) \propto\left(1-\gamma_{5}\right)(1-\gamma . \epsilon) v(p) .
\end{aligned}
$$

The appearance of the solutions of the Dirac equation for particles and antiparticles is important as it allows one to make some relationship with its achievements. The presence of the front factors, $\left(1 \pm \gamma_{5}\right)$, is essential with respect to the present developments. It shows that parity violation could be built in the description of the particles themselves. The standard model of electro-weak interactions would thus be the effective one accounting for the above feature.

The toy model considered in this section casts also some light on the currents and the appearance of the charge conjugation as an internal parity operation. Considering matrix elements of the axial current with solutions for particles and antiparticles, eq. (5), and integrating over $\epsilon^{\mu}$ together with the further assumption $\epsilon \cdot w=0$ (decoupling of the spin carried by the chiral structure and the spin of the particle as a whole, $w$ ), one gets for a particle and its antiparticle:

$$
\begin{array}{r}
<S=\frac{1}{2}, p\left|J_{\mu}^{A}(0)\right| S=\frac{1}{2}, p>=\quad \bar{u}(p) \gamma_{\mu} u(p), \\
\text { and }-\bar{v}(p) \gamma_{\mu} v(p) .
\end{array}
$$

The apparent change in the parity between the l.h.s. and r.h.s. could be disturbing but it is noticed that the current at the r.h.s. changes sign under a charge operation so that the behavior of both sides under the combined parity operation, $\mathcal{P}=P C$, is the same. This is a consequence of a charge conjugation operation generated dynamically as a parity operation on an internal chiral substructure. On the other hand, the appearance of a vector current at the r.h.s. solves the major problem of the current conservation that an axial current would raise unavoidably.

\section{Conclusion and outlook}

We presented a scheme where particles and antiparticles could be associated to the parity partners of a chiral symmetry realized in the Wigner-Weyl mode. This provides a natural explanation for parity violation in physics. We describe how charge conjugation, which is no more than a parity operation on a substructure with a chiral character, emerges in this picture. Some of the questions and problems that could be raised have been outlined.

There is far from the present sketch to a realistic theory and it is not clear whether one can deal with some constraints on combining space-time and internal symmetries [9]. We nevertheless believe that some of the ideas considered here could cast some new light in developping theories. As examples, we notice that there is no point to restore the usual parity symmetry, its violation being intrinsically tied to the chiral substructure underlying some "charges". There is no need for introducing right-handed currents in the simplest case (see remark on $S U(2)_{R}$ ), and the difference between $V$ and $A$ currents could vanish at some point. Despite their speculative character, we guess it was appropriate to remind these ideas in this year where one celebrates 50 years of parity violation.

\section{References}

1. T.D. Lee and C.N. Yang, Phys. Rev. 104, 254 (1956).

2. C. S. Wu et al., Phys. Rev. 105, 1403 (1957).

3. R.N. Mohapatra and J.C. Pati, Phys. Rev. D11, 2558 (1975).

4. L. Landau, Nucl. Phys. 3, 127 (1957).

5. T.D. Lee and G.C. Wick, Phys. Rev. 148, 1385 (1966).

6. J.H. Christensen et al., Phys. Rev. Lett. 13, 138 (1964).

7. Y. Nambu and G. Jona-Lasinio, Phys. Rev. 122, 345 (1961).

8. B. Desplanques, AIP Conference Proceedings 539, p. 130.

9. S. Coleman and J. Mandula, Phys. Rev. 159, 1251 (1967). 